\title{
Complete eradication of chronic lymphocytic leukemia with unusual skin involvement of high mitotic index after 12 cycles of Venetoclax/obinutuzumab
}

Maria Dimou ${ }^{1}$, Theodoros Iliakis ${ }^{1}$, Vasileios Pardalis ${ }^{1}$, Aikaterini Bitsani ${ }^{1}$, Marie-Christine Kyrtsonis $^{1}$, and Panayiotis Panayiotidis ${ }^{1}$

${ }^{1}$ National and Kapodistrian University of Athens School of Health Sciences

January 17, 2021

\begin{abstract}
A 51-year male patient presented with large skin masses of the chest wall that were pathologically proven to be chronic lymphocytic leukemia/small lymphocytic lymphoma(CLL/SLL) with Ki67:70\%. The patient was treated with the time-limited Venetoclax/Obinutuzumab combination for 12 cycles and CLL/SLL including the skin component was set in complete remission.
\end{abstract}

\section{Clinical Image}

Complete eradication of chronic lymphocytic leukemia with unusual skin involvement of high mitotic index after 12 cycles of Venetoclax/obinutuzumab

Maria Dimou, Theodoros Iliakis, Vasileios Paradalis, Aikaterini Bitsani, Marie-Christine Kyrtsonis, Panayiotis Panayiotidis

First Department of Internal Medicine, Propaedeutic, Hematology Clinical Trial Unit, Laiko General Hospital, Athens, Greece

Corresponding author:

Maria Dimou MD, PhD

First Department of Internal Medicine, Propaedeutic, Hematology Clinical Trial Unit, Laiko General Hospital, Athens, Greece

16 Sevastoupoleos Str, PO: 11526

Tel: +302132060981

Fax: +302132060965

e-mail: msdimou@gmail.com

\begin{abstract}
A 51-year male patient presented with large skin masses of the chest wall that were pathologically proven to be chronic lymphocytic leukemia/small lymphocytic lymphoma(CLL/SLL) with Ki67:70\%. The patient was treated with the time-limited Venetoclax/Obinutuzumab combination for 12 cycles and CLL/SLL including the skin component was set in complete remission.
\end{abstract}

Key Clinical Message 
The novel time-limited combinations with the bcl-2 inhibitor Venetoclax can induce deep responses even in CLL cases with unusual and biologically aggressive presentations, like the skin masses of our patient.

Keywords: CLL/SLL, Venetoclax, time-limited therapy, skin nodules, MRD negativity

\section{Case Presentation}

A 51-year-old male patient presented with skin masses, $10 \mathrm{~cm} \mathrm{X} 5 \mathrm{~cm}$ (figure 1), in May 2019. Biopsy showed Chronic Lymphocytic Leukemia/Small Lymphocytic Lymphoma (CLL/SLL): diffuse infiltration by small lymphocytes with the immunophenotype(IF) of CD5+, CD23+, CD79a+>CD20+. Ki67\% expression was $70 \%$. Mild lymphocytosis (lymphocytes $=13910 / \mathrm{mm}^{3}$ ) and lymphadenopathy without splenomegaly/hepatomegaly in computed tomography $(\mathrm{CT})$ scans were also present. CLL was IgVH unmutated and deletion 17p/TP53 mutations were negative. The patient was treated with the novel time-limited combination of Venetoclax/Obinutuzumab ${ }^{1}$ from July 2019 until July 2020. The CLL skin component responded impressively (figure 2 and figure 3). Complete remission in CT scans and bone marrow biopsy was confirmed at the end of treatment. Undetectable CLL minimal residual disease in peripheral blood and bone marrow by 8-colour flow cytometry was also confirmed. CLL skin involvement is a rare clinical presentation especially at diagnosis. Ki67 $>45 \%$ is associated with more aggressive biology of Non-Hodgkin lymphomas ${ }^{2}$. Our case indicates that the novel time-limited treatment with the Venetoclax /Obinutuzumab combination can eradicate the CLL disease, even with unusual and biologically aggressive presentations.

\section{Authorship list}

Maria Dimou MD - author

Theodoros Iliakis MD, co-author

Vasileios Pardalis MD, co-author

Aikaterini Bitsani MD, co-author

Marie-Christine Kyrtsonis, co-author

Panayiotis Panayiotidis, co-author

\section{Conflict of interest}

None to declare

\section{Informed consent}

Informed consent was obtained from the patient in order to use the images of the skin lesions

Funding information

None

\section{References}

Fischer K, Othman AS, Fink AM, Tandon M, Dixon M, Robrecht S, et al. Venetoclax and Obinutuzumab in Patients with CLL and Coexisting Conditions. N Engl J Med 2019; 380:2225-2236.

Broyde A, Boycov O, Strenov Y, Okon E, Shpilberg O, Bairey O. Role and prognostic significance of the Ki-67 index in non-Hodgkin's lymphoma. Am J Hematol. 2009; 84(6):338-43.

\section{Figures Legends}

Figure 1: Skin masses at CLL diagnosis

Figure 2: Skin presentation after 6 cycles of Venetoclax-Obinutuzumab

Figure 3: Skin presentation at the end of treatment 

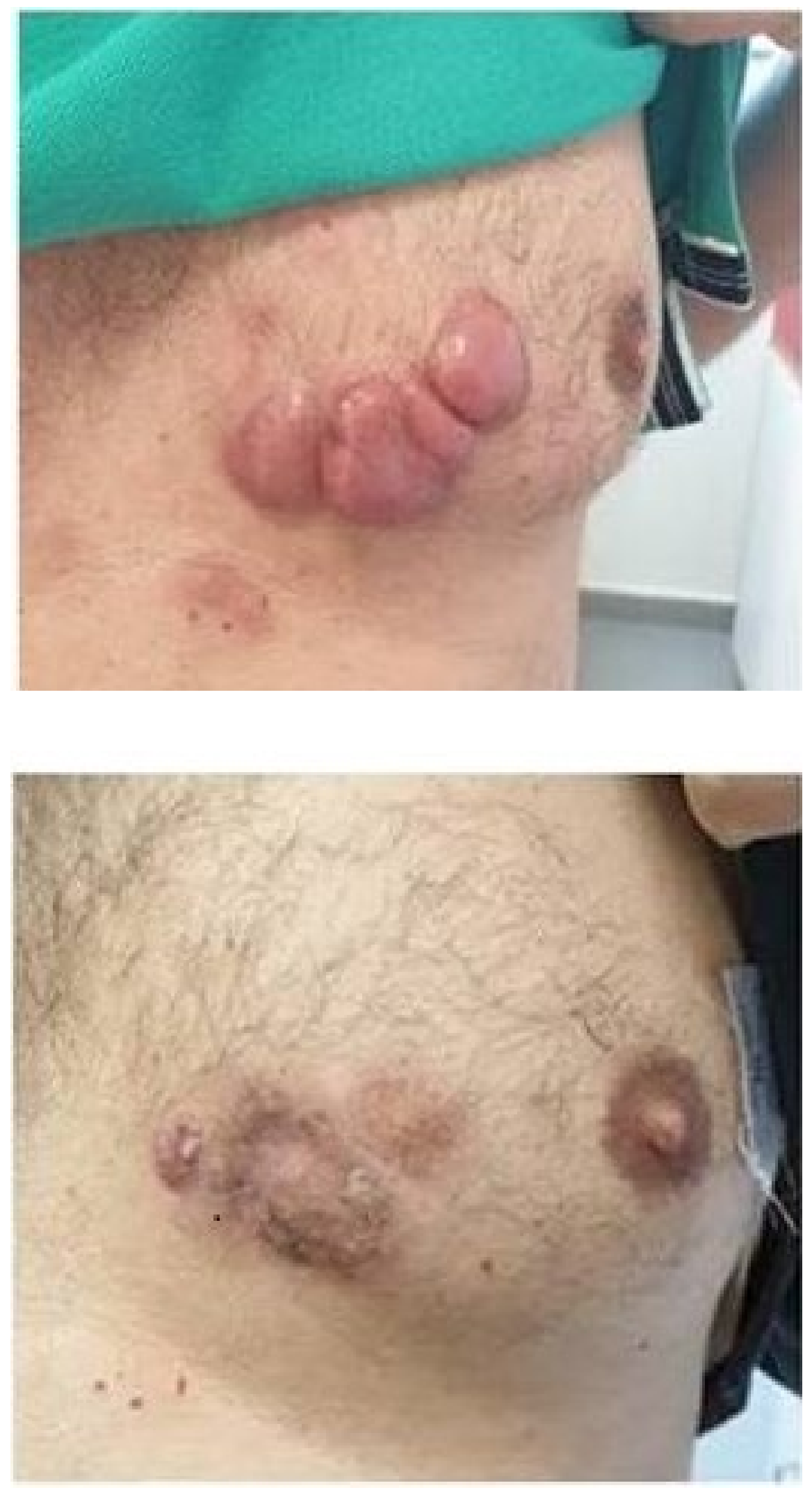


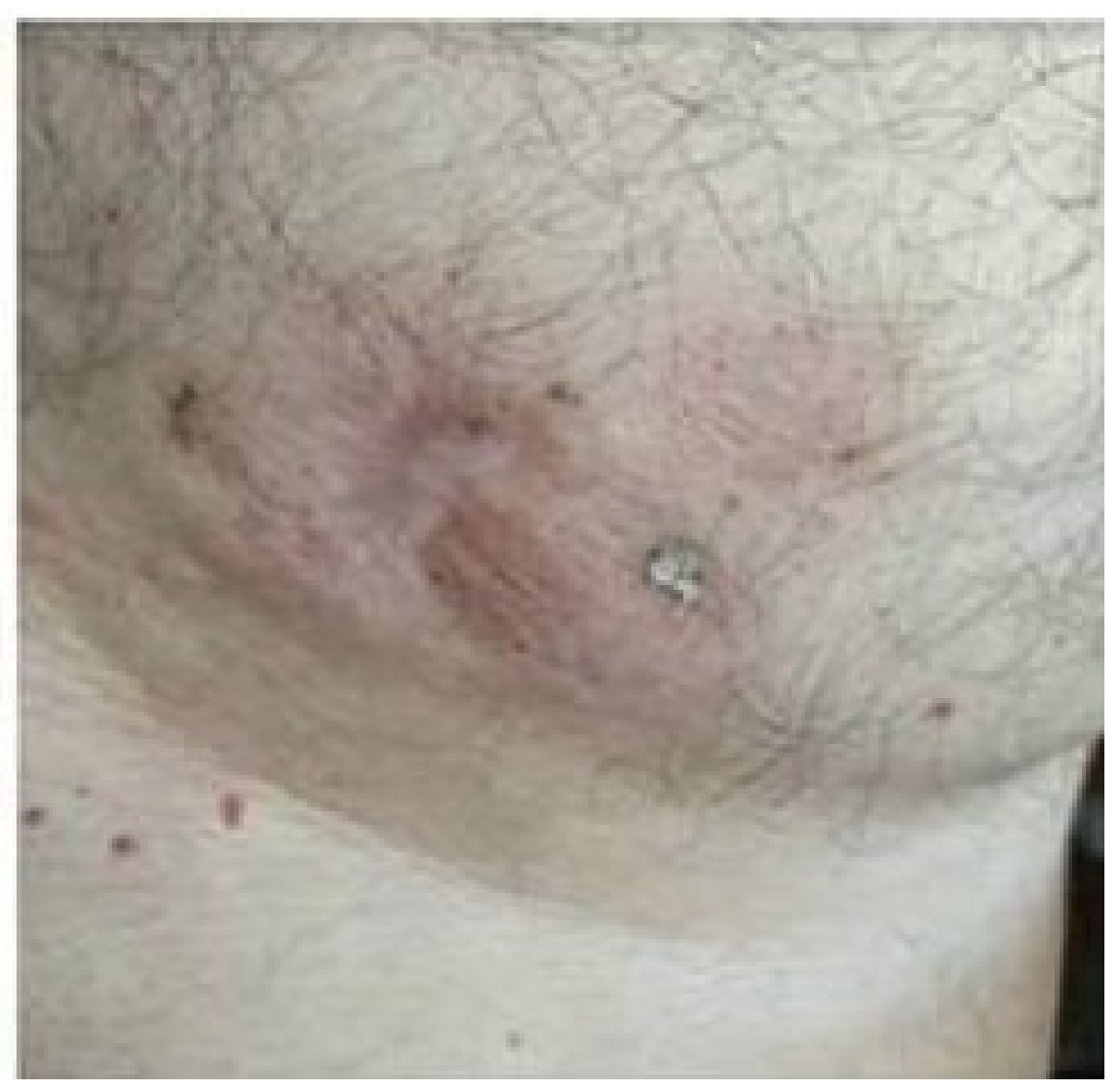

